\title{
Experimentally elevated levels of testosterone at independence reduce fitness in a territorial bird
}

\author{
J. Martínez-Padilla,,${ }^{1,7}$ L. Pérez-Rodríguez,,${ }^{1,2}$ F. Mougeot,${ }^{3,4}$ S. C. Ludwig, ${ }^{5}$ and S. M. Redpath ${ }^{6}$ \\ ${ }^{1}$ Department of Evolutionary Ecology, Museo Nacional de Ciencias Naturales (MNCN-CSIC), José Gutiérrez Abascal 2, \\ 28006 Madrid, Spain \\ ${ }^{2}$ CIBIO, Centro de Investigação em Biodiversidade e Recursos Genéticos, Campus Agrário de Vairão, Rua Padre Armando Quintas, \\ 4485661 Vairão, Portugal \\ ${ }^{3}$ Estación Experimental de Zonas Áridas (CSIC), Ctra. de Sacramento s/n, La Cañada de San Urbano, 04120 Almería, Spain \\ ${ }^{4}$ Instituto de Investigación en Recursos Cinegéticos-IREC (CSIC-UCLM-JCCM), Ronda de Toledo s/n, 13005 Ciudad Real, Spain \\ ${ }^{5}$ Game and Wildife Conservation Trust, Eggleston Hall, Barnard Castle DL120AG United Kingdom \\ ${ }^{6}$ Institute of Environmental and Biological Sciences, University of Aberdeen, Tillydrone Avenue, Aberdeen AB24 2 TZ United Kingdom
}

\begin{abstract}
Environmental conditions and individual strategies in early life may have a profound effect on fitness. A critical moment in the life of an organism occurs when an individual reaches independence and stops receiving benefits from its relatives. Understanding the consequences of individual strategies at the time of independence requires quantification of their fitness effects. We explored this period in the Red Grouse (Lagopus lagopus scoticus). In this system, testosterone and parasite (Trichostrongylus tenuis) levels are known to influence survival and reproduction, the two key components of individual fitness. We experimentally and simultaneously manipulated testosterone and parasites at three levels (high, intermediate, and control levels for both factors) in 195 young males in five populations using a factorial experimental design. We explored the effects of our treatments on fitness by monitoring reproduction and survival throughout the life of all males and estimating $\lambda_{\text {ind }}$, a rate-sensitive index of fitness. Parasite challenges increased the number of worms with a time lag, as previously found. However, we did not find significant effects of parasite manipulations on fitness, possibly because parasite abundance did not increase to harmful levels. Our hormone manipulation was successful at increasing testosterone at three different levels. Such increases in hormone levels decreased overall fitness. This was caused by reduced offspring production in the first breeding attempt rather than by any effect of the treatment on bird survival. Our results highlight that investing in high testosterone levels at independence, a strategy that might enhance short-term recruitment probability in territorial species such as Red Grouse, has a fitness cost, and can influence the resolution of the trade-off between reproduction and survival later in life.
\end{abstract}

Key words: birds; fitness; independence; Lagopus lagopus scoticus; life history; parasitism; Red Grouse; reproduction; survival; testosterone; trade-offs; Trichostrongylus tenuis.

\section{INTRODUCTION}

Environmental conditions in early life may have critical consequences for individual fitness (Lindström 1999, Metcalfe and Monaghan 2001, Lummaa and Clutton-Brock 2002). At the very early stages of life, constraining conditions may have negative consequences both in the short term and in the long term, through effects on different life-history traits (Lindström 1999, Metcalfe and Monaghan 2001, Reed et al. 2006, McGlothlin et al. 2007). One of the most critical moments in the life of an organism is when individuals become independent and stop receiving any direct or indirect benefit from their relatives (Vergara et al. 2010). Individuals at that point have to optimize the use of

Manuscript received 4 November 2012; revised 10 July 2013; accepted 16 August 2013. Final version received 13 September 2013. Corresponding Editor: K. P. Huyvaert.

7 E-mail: jmartinezpadilla12@gmail.com their own resources in order to maximize their shortterm and longer-term survival and reproduction prospects, and therefore their fitness. Despite the importance of this stage, there is a lack of experiments that manipulate the mechanisms influencing an individual's strategy at the time of independence followed by exploration of their fitness consequences.

At independence, individuals have to adjust their strategies according to the environmental context in which they live in order to maximize their fitness. For example, depending on the environmental circumstances and individual constraints, animals may have to choose between dispersing to different territories and fighting against their kin for local resources to ensure future survival or reproduction (Forero et al. 1999, Maccoll et al. 2000, Ona et al. 2002, Schaub and Hirschheydt 2009, Péron et al. 2010). Physiological and behavioral mechanisms are at the heart of these life-history tradeoffs, as reproduction and survival are often under 
hormonal control (Adkins-Regan 2005, Hau 2007). Testosterone in males is a key hormonal mechanism that allows individuals to adjust their sexual and territorial behavior to their surrounding environment (Stearns 1992, Sinervo and Svensson 1998, Ketterson and Nolan 1999, Adkins-Regan 2005). However, maintaining high testosterone levels has associated costs, and males have to trade off investing in testosteronedependent behavior against other life-history traits (Reed et al. 2006) and self-maintenance (Folstad and Karter 1992). Thus, the costs of maintaining high testosterone levels in early life may have critical fitness consequences later in life (Reed et al. 2006).

One proximate cost of maintaining high levels of testosterone is an increased risk of parasitism (Hamilton and Zuk 1982, Folstad and Karter 1992). Parasites have multiple physiological effects (Horak et al. 2004, Martínez-Padilla et al. 2010, Mougeot et al. 2010), resulting in a negative influence on survival or reproduction (Hudson 1986a, Martínez-de la Puente et al. 2010). Interactions between testosterone and parasites are known to operate in opposite directions: immune responses triggered by parasites can limit testosterone levels (Boonekamp et al. 2008) and the immunosuppressive effects of testosterone can increase parasite infection levels (Folstad and Karter 1992, Saino et al. 1995, Uller and Olsson 2003, Mougeot et al. 2004; but see Roberts et al. 2004, Seivwright et al. 2005). As such, variation in investment in testosterone-mediated behavior at independence may influence the risk of parasitism and vice versa, thereby altering life-history trade-off resolutions and, ultimately, fitness.

Quantifying how levels of testosterone and parasites at independence affect individual fitness requires an experiment. In this study, we manipulated testosterone and parasite levels at independence and measured subsequent fitness in male Red Grouse (Lagopus lagopus scoticus). The Red Grouse is a game bird inhabiting the heather (Calluna vulgaris) moorlands of the United Kingdom. Males establish territories in autumn and maintain them over winter (Watson and Moss 1988, 2008, Moss et al. 1996, Moss and Watson 2001). In spring, successful territorial males pair up, with those in better condition and displaying bigger sexual ornaments being more likely to obtain a mate (Mougeot et al. $2005 d$, Redpath et al. 2006b). In early spring, when pairing starts, males defend their territory along with the females. Grouse chicks leave the nest soon after hatching and both adult males and females care for their broods until the autumn, when young reach independence (Mougeot et al. 2005a, c, Watson and Moss 2008). Obtaining a territory is essential for males, especially so for young males, because survival prospects of nonterritory holders over winter are very low (Watson 1985, Watson and Moss 2008). Aggressive birds are more likely to hold a territory and male aggressiveness is affected either by increased levels of testosterone (Mougeot et al. 2003b, 2005a) or by decreased parasit- ism levels (Fox and Hudson 2001, Mougeot et al. $2005 b$ ). In addition, reproductive success is positively related to testosterone levels in males in the previous autumn (Redpath et al. 2006b). This variation in aggressive behavior throughout the Red Grouse life cycle is accompanied by seasonal variations in testosterone levels, which show a low peak in in autumn and a higher peak in spring (Mougeot et al. 2005a). Seasonal changes in testosterone levels during autumn do not differ between adult males and yearlings that have to establish a territory in their first winter of life (Mougeot et al. 2005a, Vergara and Martinez-Padilla 2012).

The main parasite of Red Grouse is the intestinal nematode Trichostrongylus tenuis, which reduces survival, productivity, and sexual displays (Hudson 1986a, Redpath et al. 2006a, Martínez-Padilla et al. 2007, 2010, Mougeot et al. 2009, 2010). Testosterone and parasites do not act independently; previous experiments have shown that testosterone affects parasitism risk (Mougeot et al. 2005a, Seivwright et al. 2005, Bortolotti et al. 2009), and that parasites affect aggression levels (Fox and Hudson 2001, Mougeot et al. 2005b). Moreover, both factors exert an interactive effect over different physiological indicators in male Red Grouse (Mougeot et al. 2007, 2009, Bortolotti et al. 2009, Martínez-Padilla et al. 2010). Thus, the Red Grouse- $T$. tenuis system is particularly well-suited for exploring the effect of testosterone and parasite levels on individual fitness.

We captured 195 yearling Red Grouse males in five populations during independence in autumn (Mougeot et al. 2003b). We experimentally manipulated testosterone and $T$. tenuis nematode parasite abundance at three levels (control, moderate, or large increases) in both variables using a factorial experimental design. We then fitted birds with radio transmitters, recaptured all birds in subsequent autumns and springs, and measured survival and productivity. We predicted (1) that an experimental increase of testosterone would be beneficial in the following breeding season (Mougeot et al. 2004, $2005 a$, Redpath et al. 2006b), but not over the course of a lifetime (Seivwright et al. 2005), resulting in a cost paid in terms of reduced lifetime fitness; (2) that increased parasite levels would have a negative effect on fitness (Hudson 1986a, Bortolotti et al. 2009, Mougeot et al. 2009, 2010), and (3) that there would be an interactive effect, such that the cost of elevated testosterone on fitness would be increased by parasites.

\section{Material and Methods \\ Catching and tagging}

In autumn 2007 and $2008\left(\mathrm{~S}_{1}, 26\right.$ September \pm 7 days; Fig. 1; Appendix A: Fig. A1) we caught 195 juvenile males by spotlighting and netting them at night on five upland estates (moors), three in England (Moorhouse, Geltsdale, and Catterick; MH, GE, and CT, respectively) and two in Scotland (Edinglassie and Invermark; EG and IM, respectively). Birds were individually ringed and fitted with a radio collar with a unique frequency 


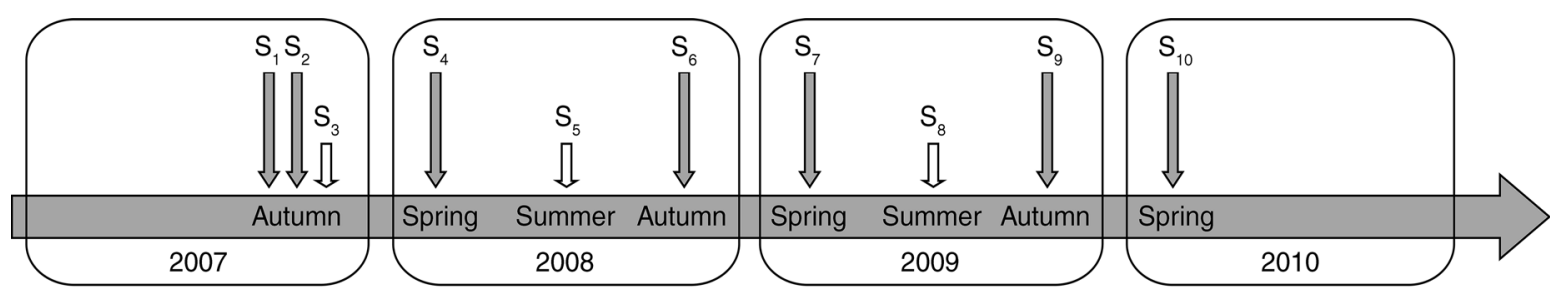

Experimental treatments by time step

$\mathrm{S}_{1}$

Dosed with anthelmintic

Testosterone treatment:

$T_{0}$, No testosterone

$T_{1}$, Intermediate levels of testosterone

$T_{2}$, High levels of testosterone
$\mathrm{S}_{2}$

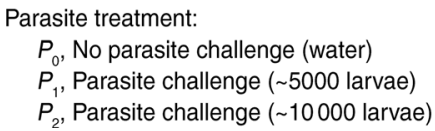

FIG. 1. Chronogram of the experiment with Red Grouse, Lagopus lagopus scoticus; $\mathrm{S}_{t}$ denotes sampling (S) at a given time ( $t$ ). Longer gray vertical arrows show the time steps when birds were captured. Shorter white vertical arrows indicate when birds were located, but not captured, during breeding. At $\mathrm{S}_{3}$, birds were only located.

(TW3-necklace radio tags; Biotrack, Dorset, UK) to facilitate relocation and recapture in the field. Each was monitored for up to three years. We assumed that this monitoring represented a reliable estimate of Red Grouse life span (see Appendix A for further details).

We located each individual up to 10 times (Fig. 1). However, we only captured each individual up to seven times, because at $\mathrm{S}_{5}$ and $\mathrm{S}_{8}$ we simply located and flushed individuals to record breeding output, breeding success, and mating status. Upon each capture, males were kept overnight in individual boxes to collect fecal samples used for estimating $T$. tenuis nematode parasite abundance. However, given that we were unable to collect fecal samples from all birds, we did not have enough sample size to test the effect of parasite treatment from $\mathrm{S}_{7}$ onward. Upon the first capture only, all birds were given a $1-\mathrm{mL}$ dose of the anthelminthic Levamisole hydrochloride (Nilverm GoldTM, ScheringPlough Animal Health, Welwyn Garden City, UK) in the morning before release. This anthelminthic dose is effective at purging grouse of their adult $T$. tenuis nematodes (Hudson 1986a, Redpath et al. 2006a, Martínez-Padilla et al. 2007, 2012). Upon each capture, we also took a blood sample to assess testosterone levels. Blood samples were collected on site at the time of capture and were kept refrigerated before they were centrifuged. Plasma and pellet samples were stored after centrifugation in a freezer at $-20^{\circ} \mathrm{C}$ during the fieldwork period and then kept at $-80^{\circ} \mathrm{C}$ until analyzed in the lab.

\section{Experiment}

All males were randomly assigned to one of the three levels of testosterone $\left(T_{\mathrm{t}}\right.$; testosterone-treated birds) and one of the three levels of parasite $\left(P_{\mathrm{t}}\right.$; parasite-treated birds) treatments, using a factorial experimental design. Hormone-treated birds were randomly assigned to one of three testosterone levels: sham-implanted birds $\left(T_{0}\right)$, intermediate levels of testosterone $\left(T_{1}\right)$, and higher levels of testosterone $\left(T_{2}\right)$. Two weeks after dosing males with the anthelminthic, males were recaptured again and parasite-treated birds were randomly assigned to no parasite challenge $\left(P_{0}\right)$, intermediate levels of parasites $\left(P_{1}\right)$, and higher levels of parasites $\left(P_{2}\right)$. As a result, all birds were assigned to one of the nine resulting treatments (see Appendix A: Table A1 for $T$ and $P$ doses and other details).

Testosterone treatments and assays.-Males were implanted with two silastic tubes $(1.57 \mathrm{~mm}$ inner and $2.41 \mathrm{~mm}$ outer diameter each) sealed with glue at both ends that varied in length depending on the testosterone treatment. $T_{1}$ - and $T_{2}$-treated birds were given two implants filled with testosterone (Sigma Aldrich Ltd, Poole, Dorset, UK) of $10 \mathrm{~mm}$ and $20 \mathrm{~mm}$ length, respectively. $T_{0}$-treated birds were given two $10 \mathrm{~mm}$ long empty implants. Implants were inserted subcutaneously between the skin and left breast muscle on the flank, under local anesthesia. We previously determined the length of the tubing during trials on captive grouse so that implants would last for 2-3 months (F. Mougeot and J. Martínez-Padilla, unpublished data), i.e., during the autumn territorial contests.

We measured plasma testosterone concentration using a commercially available testosterone enzyme immunoassay (ELISA Kit EIA-1559 from DRG Diagnostics, Marburg, Germany), which has been developed and validated for determining testosterone levels in small volumes of avian plasma $(25 \mu \mathrm{L})$. Coefficients of variation intra- and inter-assays were $3.34 \%$ and $8.26 \%$, respectively. The detection limit was $0.2 \mathrm{ng} / \mathrm{mL}$. Duplicate sample analyses showed that the testosterone assays were highly repeatable $\left(F_{25,62}=19.13, P<0.001\right.$; $r=0.93)$.

Parasite treatments. - For the parasite treatments, $P_{0^{-}}$ males were given $1.5 \mathrm{~mL}$ of water, $P_{1}$-challenge males were given an oral dose of $1.5 \mathrm{~mL}$ of water containing $\sim 5000$ T. tenuis infective larvae, and $P_{2}$-males were given $1.5 \mathrm{~mL}$ of water containing $\sim 10000 T$. tenuis 
infective larvae previously cultivated in the lab (details follow; see Fig. 1).

\section{Parasite challenges and abundances}

Trichostrongylus tenuis is a main nematode parasite of Red Grouse, typically infecting all adult birds (Hudson $1986 b$ ). It has a direct life style and no alternative hosts within the same habitat (Hudson 1986b). Eggs laid by adult $T$. tenuis worms are voided onto the moor via the host's fecal droppings (Shaw et al. 1989). There, they develop into infective larvae and are ingested by grouse that are feeding on heather Calluna vulgaris (Shaw et al. 1989, Martinez-Padilla et al. 2012). We used the remains of fecal samples collected upon first capture to cultivate $T$. tenuis infective larvae for the subsequent challenges. We followed the methods for cultivation, storing, and counting $T$. tenuis larvae described in previous works (Shaw and Moss 1989, Seivwright et al. 2004, Watson and Moss 2008). To estimate T. tenuis abundance for each male, we used fecal egg concentrations, which reliably estimate numbers of worms per grouse (see Seivwright et al. 2004).

\section{Lifetime monitoring}

Because of the experimental design, we captured all individuals twice in the first autumn to test the effectiveness of the initial testosterone treatments. Also, during the first autumn, all individuals were located a third time $\left(\mathrm{S}_{3}\right)$ but not captured. Subsequently, individuals were captured every autumn and spring until spring 2010 (see Appendix A: Table A1 for further details on sample sizes by treatment).

In summer (late July-early August), grouse broods stay together until the start of the grouse hunting season (12 August) when families start breaking up (Hudson $1986 b)$. At that time $\left(\mathrm{S}_{5}\right.$ and $\mathrm{S}_{8}$; Fig. 1$)$, we counted the number of offspring raised by individual males as a measure of breeding output, a reliable method in Red Grouse to estimate offspring productivity (Redpath et al. 2006a, b, Watson and Moss 2008). Additionally, we considered breeding success (reproduced or not) as the probability of breeding successfully and mating status (mated or not). As such, a given male can be mated but have zero breeding success if, for example, the female was lost to a predator. Overall, we located (captured or not) birds up to 10 times (see Fig. 1) to estimate survival rates and reproductive variables. At each event, we recorded whether or not they were alive. Specifically, we had 10 location events $\left(S_{1}-S_{10}\right)$ for 195 males and 1007 location events overtime overall. On 89 (8.8\%) occasions, we had a successful location of a bird after it had been considered missing.

\section{Fitness estimation}

We used rate-sensitive lambda $\left(\lambda_{\text {ind }}\right)$ as an index of fitness (Brommer et al. 2002, Brommer et al. 2004) for each male. Instead of previous rate-insensitive methods (LRS, lifetime reproductive success), $\lambda_{\text {ind }}$ combines two fitness elements: reproduction and the time of reproduction. However, both parameters are highly correlated in Red Grouse (see Appendix B for further details). We calculated $\lambda_{\text {ind }}$ as a proxy of lifetime fitness as follows:

$$
1=\sum_{x} \frac{1}{2} f_{x} \lambda_{\text {ind }}^{-x}
$$

where $x$ is the age of the individual and $f_{x}$ is the number of offspring produced. Offspring production is scaled by the parent-offspring relatedness of 0.5 (McGraw and Caswell 1996). We calculated $\lambda_{\text {ind }}$ based on counting young birds $f_{x}$ as offspring before dispersal: $\lambda_{\text {ind }}$ (fledgling production). The value $\lambda_{\text {ind }}$ is the dominant eigenvalue of a matrix that has for each age $x$ the $f_{x} / 2$ values in its first row and 1 in its subdiagonal (for further details, see Appendix B; Brommer et al. 2004).

\section{Statistical analyses}

General approach.-We used SAS 9.2 for all analyses. Testosterone treatment $\left(T_{\mathrm{t}}\right)$ and parasite treatment $\left(P_{\mathrm{t}}\right)$ were included as factors (three levels each) in all models, as well as their interaction, unless stated otherwise. After seeing that intermediate and high levels of parasite challenges did not differ, we pooled $P_{1}$ - and $P_{2}$-treated birds, resulting in two levels of parasite treatments: parasite-challenged birds $(P)$ and control birds $\left(P_{0}\right.$; see Appendix A). We used pairwise comparisons to explore further significant factors or interactions. For clarity, we will specify the time step $\left(\mathrm{S}_{t}\right)$, which indicates the sampling event $(\mathrm{S})$ at time $(t)$ used for each model (see Appendix A for further details) when needed.

Treatment efficacy.-To assess treatment effects on testosterone levels, we used general linear mixed models (GLMM), using normal distribution of errors and an identity link function. Testosterone levels were logtransformed for normalization and we explored their variation between the first and second capture $\left(S_{1}-S_{2}\right)$. To explore variation in parasite abundances, we used generalized linear mixed models (GLIMMIX), using a negative binomial distribution of errors and log link function. Mixed models included individual nested within site as a random factor. We tested the effect of treatments on parasite levels at $\mathrm{S}_{1}, \mathrm{~S}_{4}$, and, $\mathrm{S}_{6}\left(T_{\mathrm{t}} \times P_{\mathrm{t}} \times\right.$ time; Fig. 1).

Fitness analyses.-We calculated $\lambda_{\text {ind }}$ in R 2.9.0 ( R Development Core Team 2009) following Brommer et al. (2002); $\lambda_{\text {ind }}$ was not normally distributed and we used Poisson distribution of errors and a log link function when it was the dependent variable in GLIMMIX models. Site was considered as a random factor.

Reproduction.-We considered three dependent variables to explore the effect of treatments on reproduction: breeding output, breeding success, and mating status. Breeding output considered the number of offspring (including zeros) that males produced. Breeding success categorized individuals in relation to their success at breeding (male that produced at least one offspring, 1; male that did not produce any, 0). Mating 
status was defined as whether a male was mated or not ( 1 , male mated; 0 , male not mated). To assess treatment effects on these variables, we used GLIMMIX models with Poisson distribution of errors and a log link function for breeding output, or with a binomial distribution of errors and a logit function for breeding success and mating status. All mixed models included the variable site as a random factor. We analyzed breeding output, breeding success, and mating success for the two breeding attempts $\left(S_{5}\right.$ and $S_{8}$; Fig. 1) altogether and separately. When included altogether, individual was nested within site as a random factor, although our experimental design did not allow us to test the interactive effect of hormone and parasite treatments over time because of sample size limitations (interaction $T_{\mathrm{t}} \times P_{\mathrm{t}} \times$ time).

Survival.-Our data failed to meet an important assumption of known fates mark-recapture approaches: independence of samples. In our case, males were from five different populations and we did not consider them to be independent samples. Given that $91.2 \%$ of the locations were successful, we assumed that we knew the fate of all individuals and analyzed survival using GLIMMIX models. We explored variation in survival probabilities in relation to parasite and testosterone treatments, using a binomial distribution of errors and a logit function. Survival (1, alive; 0, dead) was the dependent variable and testosterone treatment $\left(T_{\mathrm{t}}\right)$, parasite treatment $\left(P_{\mathrm{t}}\right)$, or their interaction were considered as factors. Site was included as a random factor in all models. We coded the life history of each bird as follows. If a bird was missing at $\mathrm{S}_{t}$ but was located later alive at $\mathrm{S}_{t+1}$, we assumed that the individual was alive at $S_{t}$. For example, if a male was first captured at $S_{1}$, located at $S_{2}$, missing at $S_{3}$, but located at $S_{4}$ and $S_{5}$, and found dead in $S_{6}$, we considered that he was alive from $S_{1}$ to $S_{5}$ and dead from $S_{6}$ onward. We considered birds as dead at the location event when we found the dead animal. If an individual was missing and did not show up anymore, it was considered missing from that point onward. For example, if a male was located at $S_{1}$, $\mathrm{S}_{2}, \mathrm{~S}_{3}$, and never located again, we considered that particular male was alive up to $S_{3}$ but missing from $S_{4}$ onward. Males missing from a given location event and never found again were excluded from the analysis from the last location event onward.

Limitation in sample sizes among experimental groups and between location events did not allow us to run time-dependent models of survival. Thus, we calculated survival probabilities at given time steps. We obtained survival probabilities at first breeding survival $\left(\mathrm{S}_{5}\right)$, second breeding survival $\left(\mathrm{S}_{8}\right)$, annual survival $\left(\mathrm{S}_{6}\right)$, and overall survival $\left(\mathrm{S}_{10}\right)$.

\section{RESULTS}

\section{Effectiveness of parasite and testosterone treatments}

Testosterone treatments influenced changes in circulating levels of testosterone during the first autumn

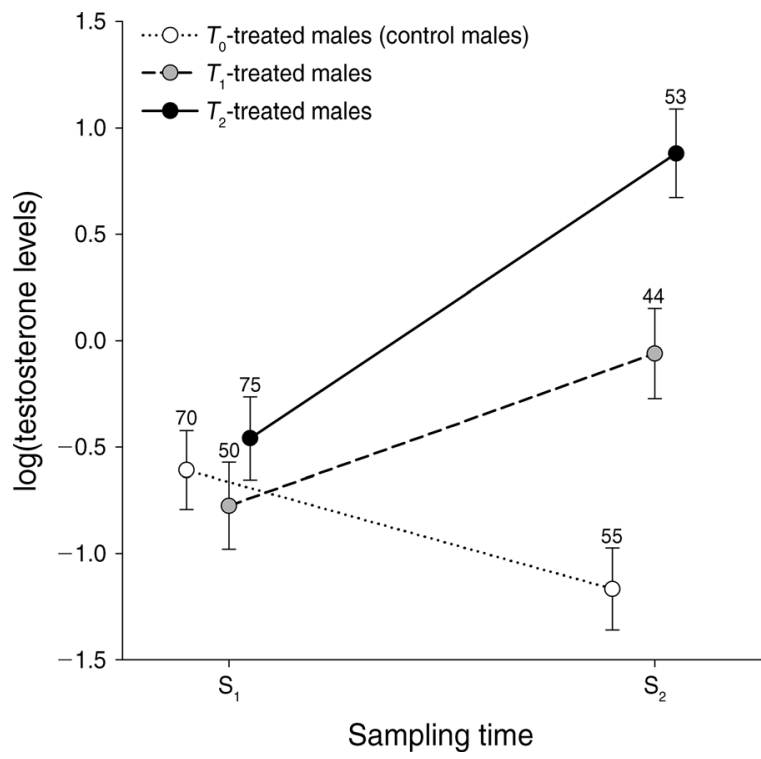

FIG. 2. Log-transformed testosterone levels (mean $\pm \mathrm{SE}$; originally measured as $\mathrm{ng} / \mathrm{mL}$ ) before $\left(\mathrm{S}_{1}\right)$ and one month after $\left(\mathrm{S}_{2}\right)$ testosterone implants in male Red Grouse during the first autumn (i.e., initial manipulation; see Appendix A for further details). White, gray, and black circles denote control males $\left(T_{0}\right)$, males implanted with intermediate levels of testosterone $\left(T_{1}\right)$, and males implanted with high levels of testosterone $\left(T_{2}\right)$, respectively. Numbers above bars denote sample size.

$\left(\mathrm{S}_{1}-\mathrm{S}_{2} ; T_{\mathrm{t}} \times\right.$ time, $\left.F_{2,92}=14.51, P<0.001\right)$. Specifically, $T_{1^{-}}$and $T_{2}$-treated birds had higher increases in testosterone levels than control birds (for $T_{0}-T_{1}$, $F_{1,65}=14.64, P<0.001$; for $T_{0}-T_{2}, F_{1,65}=27.71, P<$ $0.001)$. Furthermore, changes in levels of testosterone differed between $T_{1}$ - and $T_{2}$-treated birds, with greater increases for $T_{2}$-implanted birds than for $T_{1}$-implanted birds (for $T_{1}-T_{2}, F_{1,54}=14.64, P=0.013$; Fig. 2).

Upon initial capture $\left(\mathrm{S}_{1}\right)$, males had on average $761 \pm$ 62 worms (mean \pm SD). One month after parasite purging (dosing with anthelminthic, at $\mathrm{S}_{2}$ ), birds had 31 \pm 3 worms (mean $\pm \mathrm{SD}$ ), confirming that the purging had been effective. Parasite challenges did not increase parasite levels until one year after manipulation (upon $\mathrm{S}_{6}$; Fig. 3), for both $P_{1^{-}}$and $P_{2}$-treated birds, and at lower levels than anticipated $\left(\mathrm{S}_{6}\right.$, comparison $P_{1}-P_{0}: \chi^{2}$ $=4.10, P=0.043 ; P_{2}-P_{0}: \chi^{2}=5.64, P=0.017$; previous sampling times: all $P>0.308$ ). Moreover, parasite abundance at $\mathrm{S}_{6}$ did not significantly differ between $P_{1^{-}}$and $P_{2^{-}}$-treated birds $\left(\chi^{2}=0.32, P=0.570\right.$; Fig. 3 ), suggesting that our initial parasite treatment did not create three levels of parasites burdens, but rather two groups: unchallenged males $\left(P_{0}\right)$ and parasite-challenged males $\left(P_{1}\right.$ - and $P_{2}$-birds).

\section{Effects of testosterone and parasite treatments on fitness $\left(\lambda_{\text {ind }}\right)$}

Variation in male fitness $\left(\lambda_{\text {ind }}\right)$ depended on testosterone treatment (for $T_{\mathrm{t}}, F_{2,174}=2.75, P=0.039$; Fig. 4), but was unexplained by parasite treatment (for $P_{\mathrm{t}}, F_{1,174}$ 


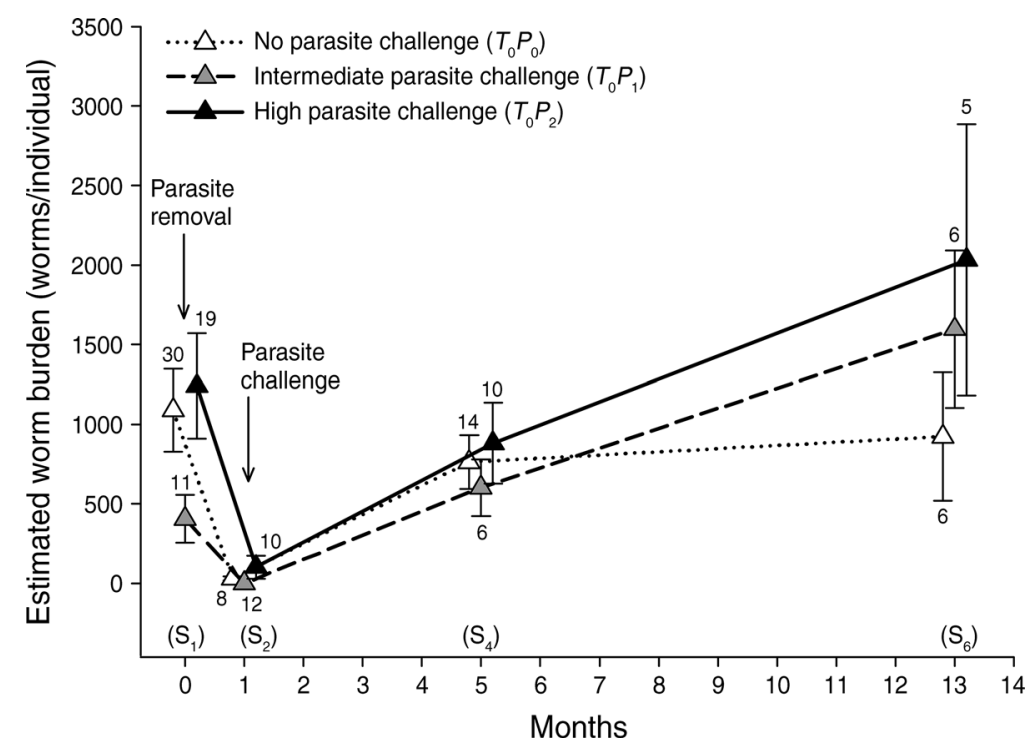

FIG. 3. Estimated Trichostrongylus tenuis worm burdens (number of worms per male, mean \pm SE) of male Red Grouse according to parasite treatment and at different sampling times. Parasite levels are shown from the start of the experiment $\left(S_{1}, S_{2}\right.$, and $\mathrm{S}_{4}$ ) until the following year $\left(\mathrm{S}_{6}\right)$, when an effect of the parasite challenges was detected. Numbers below/above error bars refer to sample size (number of males). Capture events (sampling periods) are shown in parentheses (see Fig. 1).

$=2.42, P=0.121)$ or the interaction between them $\left(H_{\mathrm{t}} \times\right.$ $\left.P_{\mathrm{t}}, F_{2,174}=0.29, P=0.751\right)$. Fitness values $\left(\lambda_{\text {ind }}\right)$ did not differ between $T_{0}$ - and $T_{1}$-treated birds $\left(F_{1,107}=0.15, P\right.$ $=0.882)$, but differed between $T_{0^{-}}$and $T_{2}$-treated birds $\left(F_{1,130}=2.24, P=0.026\right)$ and between $T_{1^{-}}$and $T_{2^{-}}$ treated birds $\left(F_{1,113}=2.00, P=0.047\right)$, with $T_{2}$-treated birds having the lowest fitness values (Fig. 4). We further investigated whether these fitness effects were due to differences in survival, reproduction, or both.

\section{Decomposing fitness into reproduction and survival}

Reproduction.-Overall $\left(\mathrm{S}_{5}\right.$ and $\left.\mathrm{S}_{8}\right)$, breeding success varied with testosterone treatment $\left(\chi^{2}=7.98, P=0.018\right)$, but not with parasite treatment $\left(\chi^{2}=0.02, P=0.660\right)$ or the interaction between both treatments $\left(\chi^{2}=0.10, P=\right.$ $0.951)$. Specifically, breeding success was lower for $T_{2^{-}}$ treated than for $T_{0}$-treated birds $\left(\chi^{2}=5.93, P=0.001\right)$ and for $T_{1}$-treated birds $\left(\chi^{2}=3.74, P=0.050\right)$, with no significant difference between $T_{0^{-}}$and $T_{1}$-treated birds $\left(\chi^{2}=0.04, P=0.823\right)$. Breeding output (i.e., the number of offspring raised by individual males) was marginally affected by testosterone treatment $\left(\chi^{2}=3.08, P=0.053\right)$, but not by parasite treatment $\left(\chi^{2}=0.85, P=0.356\right)$ or the interaction between treatments $\left(\chi^{2}=2.72, P=\right.$ 0.099). $T_{2}$-treated birds produced fewer offspring than $T_{1}$-treated birds $\left(\chi^{2}=3.95, P=0.017\right)$, but were similar to $T_{0}$-treated birds $\left(\chi^{2}=1.12, P=0.277\right)$. Breeding output did not differ between $T_{0}$ - and $T_{1}$-treated birds $\left(\chi^{2}=1.89, P=0.107\right.$; Fig. 5). Mating status was not influenced by testosterone treatment $\left(\chi^{2}=4.33, P=\right.$ $0.115)$, parasite treatment $\left(\chi^{2}=0.18, P=0.668\right)$, or a combination of both treatments $\left(\chi^{2}=0.25, P=0.881\right)$ when considering both breeding attempts $\left(S_{5}\right.$ and $\left.S_{8}\right)$.
We further analyzed separately both breeding success and output at the first $\left(\mathrm{S}_{5}\right)$ and second breeding attempt $\left(S_{8}\right)$. At the first breeding attempt $\left(S_{5}\right)$, we found no effect of parasites or their interaction with testosterone treatments (both $P>0.779$ ). Instead, testosterone

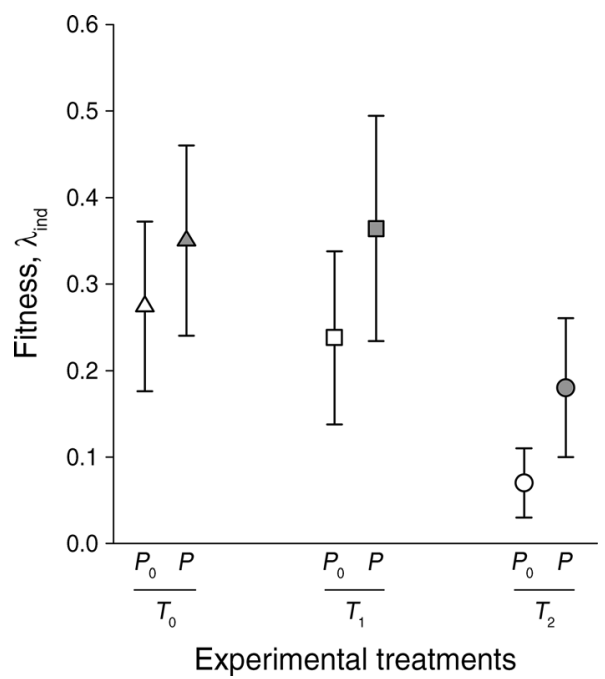

FIG. 4. Fitness $\left(\lambda_{\text {ind }}\right.$, mean \pm SE) of male Red Grouse according to testosterone and parasite treatment in early life. The rate-sensitive index of fitness of males $\left(\lambda_{\text {ind }}\right)$ was calculated following Brommer et al. $(2002,2004)$ as the number of the offspring produced during a lifetime, taking into account the time at which each individual produced its offspring. In the $x$ axis we show both testosterone (control, $T_{0}$; intermediate levels of testosterone, $T_{1}$; high levels of testosterone, $T_{2}$ ) and parasite treatments (control, $P_{0}$; parasite-challenged males, $P$ [parasitechallenged birds include $P_{1}$ - and $P_{2}$-treated birds as parasite treatment did not differ]). 
treatment had a significant effect on mating status $\left(\chi^{2}=\right.$ 5.41, $P=0.047)$, breeding success $\left(\chi^{2}=6.81, P=0.033\right)$, and breeding output $\left(\chi^{2}=8.68, P=0.013\right)$. However, the direction of the testosterone treatment effect on those reproductive variables was different. $T_{2}$-treated males had higher probabilities of being mated and breeding success than control birds (for mating status, $\chi^{2}=4.22$, $P=0.040$; for breeding success, $\left.\chi^{2}=4.18, P=0.041\right)$, but produced fewer offspring $\left(\chi^{2}=4.70, P=0.030\right) . T_{1}$ - and $T_{2}$-treated birds only differed in breeding output $\left(\chi^{2}=\right.$ $8.16, P=0.004)$ and marginally in breeding success $\left(\chi^{2}=\right.$ 3.74, $P=0.053)$, where $T_{2}$-birds produced fewer offspring and had higher probabilities of reproducing than $T_{1}$-birds. $T_{0}$ - and $T_{1}$-treated birds did not differ in any reproductive variable considered (all $P>0.088$ ). In summary, $T_{2}$-treated birds had a higher probability of being mated and of producing chicks, but produced fewer offspring than any other group. At the second breeding attempt $\left(\mathrm{S}_{8}\right)$, there were no statistically significant effects of testosterone treatment, parasite treatment, or their interactions on any reproductive variables considered (all $P>0.286$ ).

From the 195 experimental birds initially captured (Fig. 6), only five males were alive at the end of the experiment, $\mathrm{S}_{10}$ (two in IM, one in GE, one in $\mathrm{EG}$, and one in $\mathrm{MH}$; see Appendix A: Table A2). Only 55 survived to the first breeding attempt $\left(\mathrm{S}_{5}\right)$ and 16 to the second ( $\mathrm{S}_{8}$; see Appendix A). Statistical analyses did not suggest any significant effect of testosterone, parasites, or a combination of both (Table 1, Fig. 6; see Appendix $\mathrm{C}$ for further details) on any measure of survival.

\section{Discussion}

In agreement with our initial prediction, we found that birds with greater initial increase in testosterone levels ( $T_{2}$-birds) at the time of independence had lower lifetime fitness, mainly through a negative effect on reproductive output. However, this did not apply to $T_{1^{-}}$ birds, with a moderate initial increase in testosterone levels. Our parasite manipulation was not effective at increasing parasite intensities to a level at which $T$. tenuis parasites negatively affect fitness $(\sim 3000$ worms; Hudson 1986b), so it was not surprising that we did not detect significant effects of parasites on fitness, or an interactive effect of parasite and testosterone on fitness. Therefore, from our results we can only consider the effects of contrasted testosterone levels at independence on fitness in a context where parasite abundances are low.

Testosterone implants were effective at increasing testosterone levels within known natural levels for young and older males during autumn (Mougeot et al. 2009, Martínez-Padilla et al. 2010, Vergara and Martinez-Padilla 2012; see Appendix A for further details). Previous studies have found a peak in testosterone levels in autumn associated with territory defense that increases in September, peaks in October, and decreases in November (Mougeot et al. 2005a). Therefore we

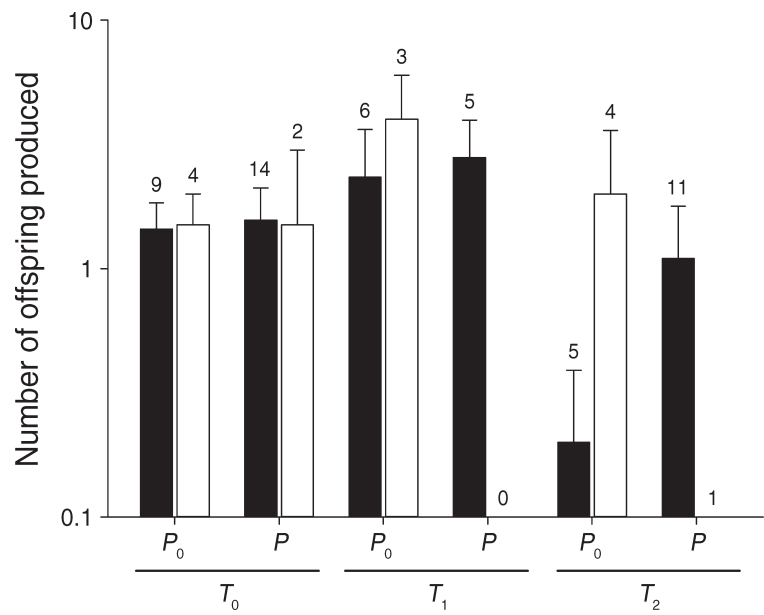

FIG. 5. Breeding output (number of offspring produced over a lifetime, mean $+\mathrm{SD}$; note the log scale) of male Red Grouse according to testosterone and parasite treatments. The $x$-axis shows both testosterone (control, $T_{0}$; intermediate levels of testosterone, $T_{1}$; high levels of testosterone, $T_{2}$ ) and parasite treatments (control, $P_{0}$; parasite-challenged males, $P$ ). Black and white bars denote first breeding $\left(\mathrm{S}_{5}\right)$ and second breeding attempt $\left(\mathrm{S}_{8}\right)$, respectively, for each treatment group. Numbers above the error bars refer to sample size (number of males).

expected equivalent similar seasonal variation in testosterone levels in our control birds. Although we found a significant increase in testosterone levels in males implanted with testosterone, we did not find an increase in control birds (see Fig. 2), possibly because the second sampling was relatively late in the autumn (end of October), at a time when testosterone levels already may have decreased naturally (Mougeot et al. 2005a).

We had no evidence that parasite treatment affected testosterone levels at any sampling time or that testosterone treatments affected parasite abundances. The negative effect of elevated testosterone levels on fitness was explained by a decrease in breeding output, because survival probabilities of testosterone-implanted birds were similar to those of control birds. Previous results in Red Grouse found weak effects of testosterone implants in autumn on male Red Grouse survival, irrespective of the bird age (Redpath et al. 2006b). Perhaps, the lack of any effect of testosterone or parasites on survival reflects the high mortality rate of wild Red Grouse, with nearly $60 \%$ dying during their first year of life. To detect survival effects in such conditions would require a much larger sample size. In addition, our initial 195 birds were split into six experimental groups across five populations, further restricting our degrees of freedom. Previous studies on Red Grouse have reported weak effects of testosterone on survival (Mougeot et al. 2005d, Redpath et al. 2006b). On the one hand, testosterone may help individuals to gain a territory and hence improve their overwinter survival, but alternatively they could pay physiological (oxidative stress, immunity) or behavioral 

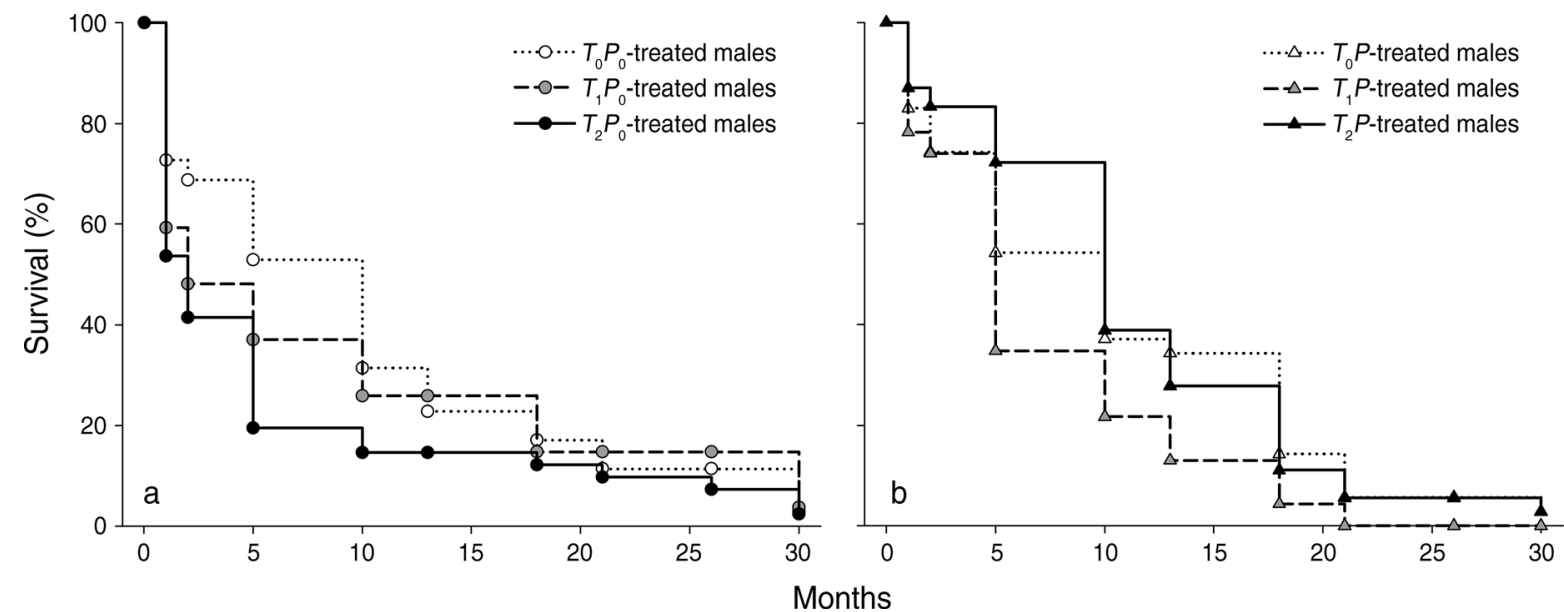

FIG. 6. Survival probabilities of young male Red Grouse over time (months) in relation to (a) hormone and (b) parasite treatments. Note that $P_{0}$ denotes parasite-free birds; $P$ denotes parasite-challenged birds and includes $P_{1}$ - and $P_{2}$-treated birds because parasite treatment did not differ between these two groups. See Results for further details.

(predation) costs of maintaining high levels of this hormone (Folstad and Karter 1992). Our results do not allow us to clearly reject the hypothesis that testosterone reduces survival, at least at the levels that we have manipulated. Independent of the mechanism, it is possible that the balance between these costs and benefits might be context-dependent. For example, different environmental conditions, like levels of intrasexual competition, may force individuals to change their resource allocation priorities (Martínez-Padilla et al. 2010, Vergara et al. 2012b), probably resulting in a context-dependent selection (Robinson et al. 2012).

Experimental increases in testosterone levels $\left(T_{2}\right)$ reduced reproductive output, principally during the first breeding attempt. In line with previous results in Red Grouse (Redpath et al. 2006b), $T_{2}$-treated males had a higher probability of finding a mate and producing offspring successfully, at least during their first mating season. However, this higher probability of forming a pair and reproducing successfully was not associated with a higher production of offspring, as it was in an earlier experiment (Redpath et al. 2006a). Here, we report the opposite. A possible explanation is that testosterone (in $T_{2}$-treated birds) had prolonged behavioral effects into their first breeding attempt, such as a greater aggressiveness (Mougeot et al. 2003b, 2005a), which reduced paternal care of broods, as suggested in Red Grouse (Moss et al. 1994) and other species (Silverin 1980). Alternatively, testosterone may have had a negative effect on sperm quality and male fertility (Turek et al. 1976, Foerster and Kempenaers 2004) and, as such, testosterone may not always have positive effects on breeding performance (McGlothlin et al. 2010). Variation in environmental conditions may help to explain this variation between findings, because testosterone may have a positive effect on breeding performance only when environmental conditions are harsh (Moss et al. 1994). The effects of high testosterone levels may be explained by the high cost that first-year males have to meet to survive; a price is later paid in terms of reduced lifetime offspring production and thus fitness.

The effects of testosterone levels on life history are controversial (Ketterson et al. 1992, Silverin 1998, Reed et al. 2006, McGlothlin et al. 2010). This controversy may arise because of the lack of knowledge of how testosterone simultaneously affects different life-history traits and, ultimately, fitness in wild conditions (CluttonBrock and Sheldon 2010). In previous studies, the effects of elevated testosterone levels on survival and reproduction were variable (Moss et al. 1994, McGlothlin et al. 2010). For instance, McGlothlin et al. (2010) showed that the maximum level of testosterone that an individual was able to raise was associated with better

TABLE 1. Estimation of time-independent survival probabilities of male Red Grouse (Lagopus lagopus scoticus) in relation to hormone (testosterone, $T_{\mathrm{t}}$ ) and parasite treatments $\left(P_{\mathrm{t}}\right)$ and their interaction.

\begin{tabular}{ccccc}
\hline \hline Survival parameter & Treatment & $F$ & $\mathrm{df}$ & $P$ \\
\hline Breeding survival $\left(\mathrm{S}_{5}\right)$ & $T_{\mathrm{t}}$ & 1.36 & 2,185 & 0.258 \\
& $P_{\mathrm{t}}$ & 1.52 & 2,185 & 0.219 \\
& $T_{\mathrm{t}} \times P_{\mathrm{t}}$ & 2.85 & 2,185 & 0.060 \\
Annual survival $\left(\mathrm{S}_{6}\right)$ & $T_{\mathrm{t}}$ & 1.37 & 2,185 & 0.257 \\
& $P_{\mathrm{t}}$ & 0.30 & 2,185 & 0.586 \\
& $T_{\mathrm{t}} \times P_{\mathrm{t}}$ & 1.82 & 2,185 & 0.165 \\
Breeding survival $\left(\mathrm{S}_{8}\right)$ & $T_{\mathrm{t}}$ & 0.10 & 2,188 & 0.908 \\
& $P_{\mathrm{t}}$ & 2.40 & 1,189 & 0.123 \\
& $T_{\mathrm{t}} \times P_{\mathrm{t}}$ & & & \\
Overall survival $\left(\mathrm{S}_{10}\right)$ & $T_{\mathrm{t}}$ & 0.25 & 2,188 & 0.781 \\
& $P_{\mathrm{t}}$ & 0.30 & 1,189 & 0.584 \\
& $T_{\mathrm{t}} \times P_{\mathrm{t}}$ & & & \\
& & & &
\end{tabular}

Notes: Estimation of survival probability for the interaction between hormone and parasite treatment is not given for the second breeding survival $\left(\mathrm{S}_{8}\right)$ and overall survival $\left(\mathrm{S}_{10}\right)$ due to sample size limitations. In all models, site was considered as random factor (see Material and methods for further details). Parameter estimates are given in Appendix C. 
survival and reproductive outcome. However, experimentally increased testosterone levels do not always increase productivity, either in the same species (Ketterson et al. 1992) or in others (Silverin 1998, Reed et al. 2006). These findings suggest that elevated testosterone levels over a threshold limit may impose a cost on individuals paid in terms of reproduction. Disparities regarding different effects of testosterone implants on life-history traits deserve further exploration, but more favorable environmental conditions (such as the size of kin groups in Red Grouse; Moss and Watson 1991, Maccoll et al. 2000, Mougeot et al. 2003b, Piertney et al. 2008) or better overall body condition of birds in a given population (Vergara et al. 2012) may help individuals to cope with the negative effect of this hormone. Within this context, higher testosterone levels at the time of independence increase aggressiveness and the likelihood of obtaining a territory to survive overwinter (Mougeot et al. 2003a, b, 2005c), but may be costly later, in terms of reduced reproductive success.

Our results agree with the idea that high testosterone levels may have a short-term positive effect (Mougeot et al. 2005d), but at the expense of a decreased reproductive success (Ketterson et al. 1992, Stearns 1992, Sinervo and Svensson 1998). We established a link between testosterone levels at the time of independence to an index of overall fitness, $\lambda_{\text {ind }}$, suggesting that high levels of testosterone are negative in terms of fitness. It implies a negative selection on those individuals with high levels of testosterone at the time of independence. Furthermore, our results allow us to distinguish between viability and fecundity selection (Garant et al. 2007, Parker et al. 2011, Potti et al. 2012). We suggest that elevated levels of testosterone at independence are under negative fecundity selection but not under viability selection in Red Grouse. Overall, elevated levels of testosterone at the time of independence might be under stabilizing selection within an evolutionary context, because those individuals are able to survive but produce fewer offspring, perhaps due to the cost of investing in a short-term survival strategy. However, further studies are required to explore the effect of environmental conditions on viability or fecundity selection in Red Grouse.

Consistent with previous work (Seivwright et al. 2005), we detected an effect of parasite challenges on parasite abundance only a year after the initial challenge. This time lag has been explained before in relation to the biology of the parasite (Hudson 1986a, $b$, Shaw and Moss 1989, Shaw et al. 1989). When conditions are unfavorable, especially in winter, larvae can arrest their development, explaining the delayed effect of parasite challenges on abundances in the host. This effect, however, was not translated into any significant effect on fitness, or any of the components of fitness that we measured. The abundance of $T$. tenuis worms in male grouse one year after the experimental challenge may have different physiological effects than those reported before (Mougeot et al. 2009, 2010), although insufficient to influence individual fitness (Hudson 1986a). This, together with the unexpectedly low infection levels caused by our challenges, might also explain the lack of interactive effect of parasites and testosterone on parasite levels, testosterone levels, reproduction, survival, or lifetime fitness.

We found that parasite burdens did not increase with increased testosterone as previously reported (Seivwright et al. 2005). This adds more controversy about a possible immunosuppressive effect of testosterone in animals (Roberts et al. 2004). Parasite levels before treatment in previous experiments in Red Grouse were much higher (2861 worms per bird; Seivwright et al. 2005) than in the present study. It is possible, therefore, that the difference can be explained by the fact that birds were in better physiological conditions because parasite levels were lower at the beginning of this experiment compared with previous studies. It is likely that birds under higher physiological stress caused by an increased intrasexual competition or higher parasitism rates may suffer chronic stress, leading to a higher susceptibility or exposure to parasites (Mougeot et al. 2005d). In fact, we have previously shown that high corticosterone levels may increase the uptake of $T$. tenuis in Red Grouse and the outcomes of parasite challenges (Bortolotti et al. 2009). This can be suggested as a potential mechanism to explain the apparent inconsistencies between previous and current results described here.

\section{Implications for population dynamics in Red Grouse}

There has been a vigorous debate about the effects of parasites and behavior on unstable population dynamics in Red Grouse (Martínez-Padilla et al. 2014). The "parasite hypothesis" invokes a destabilizing effect of $T$. tenuis, and their negative effects on female fecundity (Hudson 1986a, 1998; see also Redpath et al. 2006a). The "territorial behavior" hypothesis invokes a destabilizing effect of male aggressiveness, which regulates breeding density (Mougeot et al. 2003a). Both processes have been shown to interact: testosterone can increase parasite susceptibility (Seivwright et al. 2005) and parasites can limit aggressiveness (Fox and Hudson 2001, Mougeot et al. 2005b). The recruitment of young birds back into the population is fundamental to both hypotheses (Watson and Moss 2008). Thus, the strategy that young individuals follow to get recruited is crucial to our understanding of the mechanisms leading to unstable population dynamics. Our experiment suggests that testosterone levels influence an individual's contribution to the next generations in populations with initially low parasite abundances. Extrapolating from a life-history context to a population dynamic framework, our findings agree with the idea that high testosterone levels at the time of independence may reduce individual fitness via a lower reproductive output during their lifetime. There is little that we can say in this study about aggressiveness as the mechanism behind the territorial 
behavior hypothesis, because aggressiveness was not measured and testosterone levels are not always directly equivalent to aggressive levels (Adkins-Regan 2005, Vergara and Martinez-Padilla 2012). In addition to aggressiveness, there are several other explanations for a decrease in fitness. Assuming that high levels of testosterone mimic the behavior of young males living in populations of Red Grouse reaching their peak, we suggest that a subsequent crash in the population comes through a lower investment in reproduction due to the cost of survival. Within a broader context, our results provide strong evidence that high testosterone levels at the time of independence may have a negative effect on individual fitness and, thus, on the individual contribution of those offspring to the population.

\section{ACKNOWLEDGMENTS}

We thank the owners and gamekeepers of study sites, the British Army (Bellerby moor), RSPB (Geltsdale moor) and English Natural Heritage (Moorhouse); Chris McCarthy, David Craithness, and Derek Calder for allowing us to work in Invermark and Edinglassie, respectively. We thank $\mathrm{P}$. Vergara, D. Gil, C. Alonso-Alvarez, I. López-Rull, and J. A. Fargallo for providing very constructive comments on early versions of the manuscript; F. Casas, M. Zeinnedine, I. Pagan, J. Haines, and A. de Frutos for helping during the fieldwork; Christophe Lebigre for fitness analyses and Alex Millon for survival analyses. This study was funded by a Natural Environment Research Council grant (NE/D014352/1). J. Martínez-Padilla and L. Pérez-Rodríguez were founded by JAE-doc and JdC fellowship, respectively, from the Ministerio de Educación y Ciencia (Spain) during the writing of this manuscript. We held all the necessary UK Home Office licenses for conducting these procedures (PPL 60/3824). This study was funded by a National Environment Research Council grant (NE/D014352/1).

\section{Literature Cited}

Adkins-Regan, E. 2005. Hormones and animal social behavior. Princeton University Press, Princeton, New Jersey, USA.

Boonekamp, J. J., A. F. H. Ros, and S. Verhulst. 2008. Immune activation suppresses plasma testosterone level: a metaanalysis. Biology Letters 4:741-744.

Bortolotti, G. R., F. Mougeot, J. Martínez-Padilla, L. M. I. Webster, and S. B. Piertney. 2009. Physiological stress mediates the honesty of social signals. PLoS ONE 4:e4983.

Brommer, J. E., L. Gustafsson, H. Pietiäinen, and J. Merilä. 2004. Single-generation estimates of individual fitness as proxies for long-term genetic contribution. American Naturalist 163:505-517.

Brommer, J. E., J. Merilä, and H. Kokko. 2002. Reproductive timing and individual fitness. Ecology Letters 5:802-810.

Clutton-Brock, T., and B. C. Sheldon. 2010. Individuals and populations: the role of long-term, individual-based studies of animals in ecology and evolutionary biology. Trends in Ecology and Evolution 25:562-573.

Foerster, K., and B. Kempenaers. 2004. Experimentally elevated plasma levels of testosterone do not increase male reproductive success in blue tits. Behavioral Ecology and Sociobiology 56:482-490.

Folstad, I., and A. J. Karter. 1992. Parasites, bright males and the immunocompetence handicap. American Naturalist 139: 603-622.

Forero, M. G., J. A. Donázar, J. Blas, and F. Hiraldo. 1999. Causes and consequences of territory change and breeding dispersal in the Black Kite. Ecology 80:1298-1310.
Fox, A., and P. J. Hudson. 2001. Parasites reduce territorial behaviour in red grouse (Lagopus lagopus scoticus). Ecology Letters 4:139-143.

Garant, D., L. E. Kruuk, R. H. McCleery, and B. C. Sheldon. 2007. The effects of environmental heterogeneity on multivariate selection on reproductive traits in female great tits. Evolution 61:1546-1559.

Hamilton, W. D., and M. Zuk. 1982. Heritable true fitness and bright birds: A role for parasites? Science 218:384-387.

Hau, M. 2007. Regulation of male traits by testosterone: implications for the evolution of vertebrate life histories. BioEssays 29:133-144.

Horak, P., L. Saks, U. Karu, I. Ots, P. F. Surai, and K. J. McGraw. 2004. How coccidian parasites affect health and appearance of greenfinches. Journal of Animal Ecology 73: 935-947.

Hudson, P. J. 1986a. The effect of a parasitic nematode on the breeding production of red grouse. Journal of Animal Ecology 55:85-92.

Hudson, P. J. 1986b. The red grouse: the biology and management of a wild gamebird. The Game Conservancy Trust, Fordingbridge, UK.

Hudson, P. J., A. P. Dobson, and D. Newborn. 1998. Prevention of population cycles by parasite removal. Science 282:2256-2258.

Ketterson, E. D., and V. Nolan. 1999. Adaptation, exaptation, and constraint: a hormonal perspective. American Naturalist 154:S4-S25.

Ketterson, E. D., V. Nolan, L. Wolf, and C. Ziegenfus. 1992. Testosterone and avian life histories: effects of experimentally elevated testosterone on behavior and correlates of fitness in the Dark-Eyed Junco (Junco hyemalis). American Naturalist 140:980-999.

Lindström, J. 1999. Early development and fitness in birds and mammals. Trends in Ecology and Evolution 14:343-348.

Lummaa, V., and T. Clutton-Brock. 2002. Early development, survival, and reproduction in humans. Trends in Ecology and Evolution 17:141-147.

Maccoll, A. D. C., S. B. Piertney, R. Moss, and X. Lambin. 2000. Spatial arrangement of kin affects recruitment success in young male red grouse. Oikos 261-270.

Martínez-de la Puente, J., S. Merino, G. Tomás, J. Moreno, J. Morales, E. Lobato, S. García-Fraile, and E. J. Belda. 2010. The blood parasite Haemoproteus reduces survival in a wild bird: a medication experiment. Biology Letters 6:663-665.

Martínez-Padilla, J., F. Mougeot, L. Pérez-Rodríguez, and G. R. Bortolotti. 2007. Nematode parasites reduce carotenoid-based signalling in male red grouse. Biology Letters 3: $161-164$

Martínez-Padilla, J., F. Mougeot, L. M. I. Webster, L. PérezRodríguez, and S. B. Piertney. 2010. Testing the interactive effects of testosterone and parasites on carotenoid-based ornamentation in a wild bird. Journal of Evolutionary Biology 23:902-913.

Martínez-Padilla, J., S. M. Redpath, M. Zeineddine, and F. Mougeot. 2014. Insights into population ecology from longterm studies of red grouse Lagopus lagopus scoticus. Journal of Animal Ecology 83:85-98.

Martinez-Padilla, J., P. Vergara, F. Mougeot, and S. M. Redpath. 2012. Parasitized mates increase infection risk for partners. American Naturalist 179:811-820.

McGlothlin, J. W., J. M. Jawor, and E. D. Ketterson. 2007. Natural variation in a testosterone-mediated trade-off between mating effort and parental effort. American Naturalist 170:864-875.

McGlothlin, J. W., D. J. Whittaker, S. E. Schrock, N. M. Gerlach, J. M. Jawor, E. Snajdr, and E. D. Ketterson. 2010. Natural selection on testosterone production in a wild songbird population. American Naturalist 175:687-701. 
McGraw, J. B., and H. Caswell. 1996. Estimation of individual fitness from life-history data. American Naturalist 147:47-64.

Metcalfe, N. B., and P. Monaghan. 2001. Compensation for a bad start: grow now, pay later? Trends in Ecology and Evolution 16:254-260.

Moss, R., R. Parr, and X. Lambin. 1994. Effects of testosterone on breeding density, breeding success and survival of Red Grouse. Proceedings of the Royal Society B 258:175-180.

Moss, R., and A. Watson. 1991. Population cycles and kin selection in Red Grouse Lagopus lagopus scoticus. Ibis 133: $113-120$.

Moss, R., and A. Watson. 2001. Population cycles in birds of the grouse family (Tetraonidae). Advances in Ecological Research 32:53-111.

Moss, R., A. Watson, R. Parr, and A. R. Moss. 1996. Experimental prevention of a population cycle in Red Grouse. Ecology 77:1512-1530.

Mougeot, F., A. Dawson, S. M. Redpath, and F. Leckie. $2005 a$. Testosterone and autumn territorial behavior in male red grouse Lagopus lagopus scoticus. Hormones and Behavior 47: 576-584.

Mougeot, F., S. A. Evans, and S. M. Redpath. $2005 b$. Interactions between population processes in a cyclic species: parasites reduce autumn territorial behaviour of male red grouse. Oecologia 144:289-298.

Mougeot, F., J. R. Irvine, L. Seivwright, S. M. Redpath, and S. Piertney. 2004. Testosterone, immunocompetence, and honest sexual signaling in male red grouse. Behavioral Ecology 15:930-937.

Mougeot, F., J. Martínez-Padilla, J. D. Blount, L. PerezRodriguez, L. M. I. Webster, and S. B. Piertney. 2010. Oxidative stress and the effect of parasites on a carotenoidbased ornament. Journal of Experimental Biology 213:400407.

Mougeot, F., J. Martínez-Padilla, L. M. I. Webster, J. D. Blount, L. Pérez-Rodríguez, and S. Piertney. 2009. Honest sexual signalling mediated by parasite and testosterone effects on oxidative balance. Proceedings of the Royal Society B 276:1093-1100.

Mougeot, F., L. Pérez-Rodríguez, J. Martínez-Padilla, F. M. Leckie, and S. M. Redpath. 2007. Parasites, testosterone and honest carotenoid-based signaling of health. Functional Ecology 21:886-898.

Mougeot, F., S. B. Piertney, F. Leckie, S. Evans, R. Moss, S. M. Redpath, and P. J. Hudson. 2005c. Experimentally increased aggressiveness reduces population kin structure and subsequent recruitment in red grouse Lagopus lagopus scoticus. Journal of Animal Ecology 74:488-497.

Mougeot, F., S. M. Redpath, F. Leckie, and P. J. Hudson. 2003a. The effect of aggressiveness on the population dynamics of a territorial bird. Nature 421:737-739.

Mougeot, F., S. M. Redpath, R. Moss, J. Matthiopoulos, and P. J. Hudson. 2003b. Territorial behaviour and population dynamics in red grouse Lagopus lagopus scoticus. I. Population experiments. Journal of Animal Ecology 72:1073-1082.

Mougeot, F., S. M. Redpath, S. Piertney, and P. J. Hudson. 2005d. Separating behavioral and physiological mechanisms in testosterone-mediated trade-offs. American Naturalist 166:158-168.

Ona, A. N. D., M. G. Forero, J. A. Donázar, and F. Hiraldo. 2002. Causes and fitness consequences of natal dispersal in a population of Black Kites. Ecology 83:858-872.

Parker, T. H., T. A. Wilkin, I. R. Barr, B. C. Sheldon, L. Rowe, and S. C. Griffith. 2011. Fecundity selection on ornamental plumage colour differs between ages and sexes and varies over small spatial scales. Journal of Evolutionary Biology 24: 1584-1597.

Péron, G., J. Lebreton, and P. Crochet. 2010. Breeding dispersal in black-headed gull: the value of familiarity in a contrasted environment. Journal of Animal Ecology 79:317326.

Piertney, S. B., et al. 2008. Temporal changes in kin structure through a population cycle in a territorial bird, the red grouse Lagopus lagopus scoticus. Molecular Ecology 17:2544-2551.

Potti, J., J. A. Dávila, J. L. Tella, O. Frías, and S. Villar. 2012. Gender and viability selection on morphology in fledgling pied flycatchers. Molecular Ecology 11:1317-1326.

R Development Core Team. 2009. R 2.9.0. A language and environment for statistical computing. R Foundation for Statistical Computing, Vienna, Austria.

Redpath, S. M., F. Mougeot, F. M. Leckie, D. A. Elston, and P. J. Hudson. 2006a. Testing the role of parasites in driving the cyclic population dynamics of a gamebird. Ecology Letters 9:410-418.

Redpath, S. M., F. Mougeot, F. Leckie, and A. D. Evans. $2006 \mathrm{~b}$. The effects of autumn testosterone on survival and productivity in red grouse Lagopus lagopus scoticus. Animal Behaviour 71:1297-1305.

Reed, W. L., M. E. Clark, P. G. Parker, S. A. Raouf, N. Arguedas, D. S. Monk, E. Snajdr, V. Nolan, and E. D. Ketterson. 2006. Physiological effects on demography: a long-term experimental study of testosterone's effects on fitness. American Naturalist 167:667-683.

Roberts, M. L., K. L. Buchanan, and M. R. Evans. 2004. Testing the immunocompetence handicap hypothesis: a review of the evidence. Animal Behaviour 68:227-239.

Robinson, M. R., G. Sander van Doorn, L. Gustafsson, and A. Qvarnström. 2012. Environment-dependent selection on mate choice in a natural population of birds. Ecology Letters 15:611-618.

Saino, N., A. P. Møller, and A. M. Bolzern. 1995. Testosterone effects on the immune system and parasite infestations in the barn swallow (Hirundo rustica): An experimental test of the immunocompetence hypothesis. Behavioral Ecology 6:397404.

Schaub, M., and J. V. Hirschheydt. 2009. Effect of current reproduction on apparent survival, breeding dispersal, and future reproduction in barn swallows assessed by multistate capture-recapture models. Journal of Animal Ecology 78: $625-635$.

Seivwright, L. J., S. M. Redpath, F. Mougeot, F. Leckie, and P. J. Hudson. 2005. Interactions between intrinsic and extrinsic mechanisms in a cyclic species: testosterone increases parasite infection in red grouse. Proceedings of the Royal Society B 272:2299-2304.

Seivwright, L., S. M. Redpath, F. Mougeot, L. Watt, and P. J. Hudson. 2004. Faecal egg counts provide a reliable measure of Trichostrongylus tenuis intensities in free-living red grouse Lagopus lagopus scoticus. Journal of Helminthology 78:6976.

Shaw, J. L., and R. Moss. 1989. The role of parasite fecundity and longevity in the success of Trichostrongylus tenuis in lowdensity red grouse populations. Parasitology 99:253-258.

Shaw, J. L., R. Moss, and A. W. Pike. 1989. Development and survival of the free-living stages of Trichostrongylus tenuis, a cecal parasite of red grouse Lagopus lagopus scoticus. Parasitology 99:105-113.

Silverin, B. 1980. Effects of long-acting testosterone treatment on free-living pied flycatchers, Ficedula hypoleuca, during the breeding period. Animal Behaviour 28:906-912.

Silverin, B. 1998. Territorial behaviour and hormones of pied flycatchers in optimal and suboptimal habitats. Animal Behaviour 56:811-818.

Sinervo, B., and E. Svensson. 1998. Mechanistic and selective causes of life history trade-offs and plasticity. Oikos 83:432442 .

Stearns, S. C. 1992. The evolution of life histories. Oxford University Press, Oxford, UK. 
Turek, F. W., C. Desjardins, and M. Menaker. 1976 Antigonadal and progonadal effects of testosterone in male house sparrows. General and Comparative Endocrinology 28:395-402.

Uller, T., and M. Olsson. 2003. Prenatal exposure to testosterone increases ectoparasite susceptibility in the common lizard (Lacerta vivipara). Proceedings of the Royal Society B 270:1867-1870.

Vergara, P., J. A. Fargallo, and J. Martínez-Padilla. 2010. Reaching independence: food supply, parent quality, and offspring phenotypic characters in kestrels. Behavioral Ecology 21:507-512.
Vergara, P., and J. Martinez-Padilla. 2012. Social context decouples the relationship between a sexual ornament and testosterone levels in a male wild bird. Hormones and Behavior 62:407-412.

Vergara, P., J. Martinez-Padilla, F. Mougeot, F. Leckie, and S. M. Redpath. 2012. Environmental heterogeneity influences the reliability of secondary sexual traits as condition indicators. Journal of Evolutionary Biology 25:20-28.

Watson, A. 1985. Social class, socially-induced loss, recruitment and breeding of red grouse. Oecologia 67:493-498.

Watson, A., and R. Moss. 1988. Spacing behaviour and population limitation in Red Grouse. Auk 105:207-208.

Watson, A., and R. Moss. 2008. Grouse. Collins, London, UK.

\section{Supplemental Material}

\section{Appendix A}

Experimental design, fitness estimations, and parameter estimations (Ecological Archives E095-087-A1).

\section{Appendix B}

Fitness estimations (Ecological Archives E095-087-A2).

\section{Appendix C}

Parameter estimates of treatments in Red Grouse survival (Ecological Archives E095-087-A3). 